\title{
Modeling Cascaded Cylindrical Metasurfaces with Spatially-Varying Impedance Distribution
}

\author{
Zvonimir SIPUS ${ }^{1}$, Zoran ERES ${ }^{2}$, Dominik BARBARIC ${ }^{3}$ \\ ${ }^{1}$ Faculty of Electrical Engineering and Computing, University of Zagreb, Unska 3, 10000 Zagreb, Croatia \\ ${ }^{2}$ Rudjer Boskovic Institute, Bijenicka 54, 10000 Zagreb, Croatia \\ ${ }^{3}$ Ericsson Nikola Tesla d.d., Research and Development Centre, Krapinska 45, 10000 Zagreb, Croatia
}

zvonimir.sipus@fer.hr, zoran.eres@irb.hr, dominik.barbaric@ericsson.com

Submitted July 21, 2019 / Accepted July 29, 2019

\begin{abstract}
Modeling curved metasurface structures represents a computing challenge due to the complexity of considered designs. This creates a need for specialized efficient analysis methods. An approach that combines the spectral-domain field representation and surface sheet impedance concept is proposed. The considered cascaded cylindrical metasurface structures can span across only a part of a canonical surface and unit cell elements can vary along the metasurface, giving a spatially-varying sheet impedance. The analysis method is experimentally verified against a cylindrical metasurface for shaping the feed antenna beam. The problem of manufacturing curved metasurfaces is also discussed in the paper.
\end{abstract}

\section{Keywords}

Metasurfaces, curved metasurfaces, spectral domain approach, sheet impedance, metasurface production technology, high-frequency conductive materials

\section{Introduction}

Multilayered dielectric structures with embedded metallic patterns, commonly referred to as metasurfaces, can act as electromagnetic devices that direct EM waves, manipulate the polarization of transmitted or reflected waves, or influence the spectral properties of those waves. This enables a wider range of functionalities, such as focusing, beam tilting, polarization manipulation, and increased bandwidth and angular performance [1-3]. The building blocks of metasurfaces are sub-wavelength elements that replace traditional resonant elements previously used in the design of periodic frequency selective surfaces (FSS) [4].

Until now, most of the attention was focused on planar metasurfaces that can be realized using conventional printed circuit board (PCB) fabrication techniques. For such surfaces, efficient design approaches based on surface impedance boundary conditions (both penetrable [2], [3],
[6-11] and opaque surface impedance formulations [5], [12], [13]) have been developed. Introduction of curvature, needed either for mechanical, aerodynamic or electromagnetic reasons, significantly complicates the analysis, as one need to deal with finite dimensions and more complex periodicity. In more details, the incident plane wave is scattered into a spectrum of reflected waves, as opposed to a single plane wave produced by scattering against a planar metasurface. Further, the projection of a periodic grid onto curved surfaces becomes an issue, in particular for structures that are curved in both principal directions. Cylindrical metasurfaces, fabricated from flexible PCB substrate material, have been considered in past as an attractive class of curved metasurface structures. They were used for reshaping radiation patterns [14], [15], mantle cloak realizations [16], [17], reduction of antenna blockage [18], and scattering manipulation and camouflage [19].

Some devices, such as cylindrical or spherical cloaks, would require homogeneous distribution of surface impedance. However, many devices require spatially-varying impedance distribution in order to modulate phase and/or amplitude of incoming wave. Quite often a metasurface occupying only a part of canonical surface (such as a cylinder or a sphere) is to be designed. One example is a dome antenna used to either flatten the gain or to enhance the gain of the antenna array placed inside the dome.

The aim of this paper is to discuss the spectral-domain analysis approach of a class of metasurface structures with spatially-varying impedance distribution. The spectral-domain approach is selected as it is a traditional way of determining EM field distribution in multilayer cylindrical structures. The basis of the analysis approach for spatiallyuniform metasurfaces is described in [20]. However, there is additional freedom in selecting the domain (spectral or spatial) to be used while calculating the surface sheet impedance distribution, and selecting whether the EM field in each cylindrical layer, or the induced current distribution in each curved metasurface sheet is the quantity to be determined by the analysis. All these options will be discussed in the first part of this paper, starting with an analysis of a basic single-layer metasurface, and expanding it for gen- 
eral multilayer multi-metasurface structure formulations. This is further exploited in Sec. 3 where one design example is studied in detail, as well as the production of curved metasurface structures.

\section{Analysis Approach}

The geometry of the considered cylindrical problem is shown in Fig. 1(a). It is a multilayer metasurface structure excited by an arbitrary feed antenna. Each metasurface layer is modelled by a penetrable sheet impedance boundary condition.

The analysis method will be explained by considering the basic geometry, as seen in Fig. 1(b): a 2D single-layer metasurface which is non-homogeneous in $\phi$-direction and homogeneous in axial direction. The metasurface is located in free space, and it is excited from the central axis; i.e. the excitation is a constant current line source. Without losing generality, we will assume that the considered metasurface has electric response only. Note that it is analytically shown that bianisotropic metasurfaces can be realized by cascading anisotropic, patterned metallic sheets described by an electric sheet admittance tensor $\overline{\bar{Y}}(\phi, z)$ [2]. The boundary condition to be satisfied is the following:

$$
\overline{\bar{Y}}(\phi, z) \cdot \mathbf{E}\left(\rho_{\text {meta }}, \phi, z\right)=\hat{\rho} \times \Delta \mathbf{H}\left(\rho_{\text {meta }}, \phi, z\right)
$$

where $\Delta \mathbf{H}$ represents the magnetic field discontinuity between the outer and inner boundary of the metasurface. The considered metasurface can also be constrained to only a part of the cylindrical tube. For the rest of the tube, we then impose the following boundary condition:

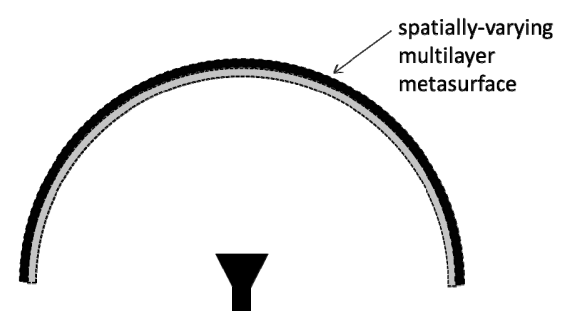

(a)

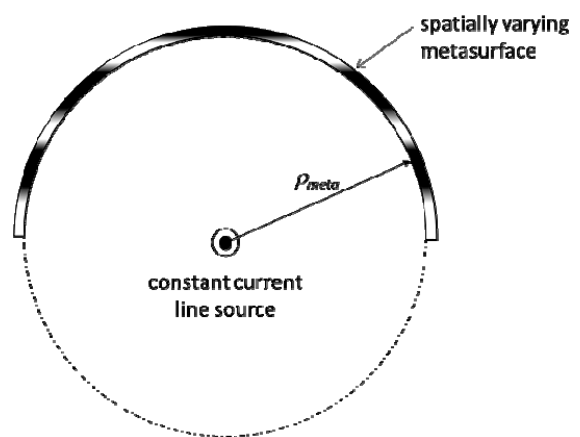

(b)

Fig. 1. Geometry of a curved metasurface structure with spatially-varying value of surface sheet impedance; (a) general multilayer metasurface with an arbitrary feed antenna, (b) basic 2D geometry.

$$
\hat{\rho} \times \Delta \mathbf{H}\left(\rho_{\text {meta }}, \phi, z\right)=0
$$

The two boundary conditions can be merged if the unpopulated part of the cylindrical surface is represented by a zero-admittance sheet. Now, the boundary condition for the whole cylindrical interface is given by (1).

The incident field excited by a constant-current line source is:

$$
\begin{aligned}
& E_{\mathrm{z}}^{\mathrm{inc}}(\rho, \phi)=-\frac{k_{0} \eta_{0}}{4} H_{0}^{(2)}\left(k_{0} \rho\right) \cdot I_{0}, \\
& H_{\phi}^{\mathrm{inc}}(\rho, \phi)=-\mathrm{j} \frac{k_{0}}{4} H_{1}^{(2)}\left(k_{0} \rho\right) \cdot I_{0} .
\end{aligned}
$$

Here, $I_{0}$ is the amplitude of the line source current. Under these assumptions we can write the field distribution in regions inside and outside the cylinder:

(a) the inner region

$$
\begin{aligned}
& E_{\mathrm{z}}^{\mathrm{tot}}=E_{\mathrm{z}}^{\mathrm{inc}}+E_{\mathrm{z}}^{\mathrm{scat}}, \\
& E_{\mathrm{z}}^{\mathrm{tot}}(\rho, \phi)= \\
& \quad-\frac{k_{0} \eta_{0}}{4} H_{0}^{(2)}\left(k_{0} \rho\right) I_{0}+\sum_{m=-\infty}^{\infty} \alpha_{m}^{(1)} J_{m}\left(k_{0} \rho\right) \mathrm{e}^{-\mathrm{j} m \phi}, \\
& H_{\phi}^{\mathrm{tot}}=H_{\phi}^{\mathrm{inc}}+H_{\phi}^{\mathrm{scat}}, \\
& H_{\phi}^{\mathrm{tot}}(\rho, \phi)= \\
& \quad-\frac{\mathrm{j} k_{0}}{4} H_{1}^{(2)}\left(k_{0} \rho\right) I_{0}+\left(\frac{-\mathrm{j}}{\eta_{0}}\right) \sum_{m=-\infty}^{\infty} \alpha_{m}^{(1)} J_{m}^{\prime}\left(k_{0} \rho\right) \mathrm{e}^{-\mathrm{j} m \phi},
\end{aligned}
$$

(b) the outer region

$$
\begin{aligned}
& E_{\mathrm{z}}^{\mathrm{tot}}=E_{\mathrm{z}}^{\mathrm{inc}}+E_{\mathrm{z}}^{\mathrm{scat}}, \\
& E_{\mathrm{z}}^{\mathrm{tot}}(\rho, \phi)= \\
& \quad-\frac{k_{0} \eta_{0}}{4} H_{0}^{(2)}\left(k_{0} \rho\right) I_{0}+\sum_{m=-\infty}^{\infty} \alpha_{m}^{(2)} H_{m}^{(2)}\left(k_{0} \rho\right) \mathrm{e}^{-\mathrm{j} m \phi}, \\
& H_{\phi}^{\mathrm{tot}}=H_{\phi}^{\mathrm{inc}}+H_{\phi}^{\mathrm{scat}}, \\
& H_{\phi}^{\mathrm{tot}}(\rho, \phi)= \\
& -\frac{\mathrm{j} k_{0}}{4} H_{1}^{(2)}\left(k_{0} \rho\right) I_{0}+\left(\frac{-\mathrm{j}}{\eta_{0}}\right) \sum_{m=-\infty}^{\infty} \alpha_{m}^{(2)} H_{0}^{(2)^{\prime}}\left(k_{0} \rho\right) \mathrm{e}^{-\mathrm{j} m \phi} .
\end{aligned}
$$

Here, $\alpha_{m}{ }^{(1)}$ and $\alpha_{m}{ }^{(2)}$ are amplitudes of scattered field's spectral components in inner and outer region, respectively. In (3)-(5), $J_{m}$ is the Bessel function, $H_{m}{ }^{(2)}$ is the Hankel function of the second kind, and $k_{0}$ and $\eta_{0}$ are the wave number and the wave impedance of free space. Note that $H_{\mathrm{z}}$ and $E_{\phi}$ components are zero for the considered metasurface structure and line source excitation. In other words, we assumed that the metasurface does not excite cross-polar components.

The boundary condition (1) can be fulfilled both in the spatial and in the spectral domain (the Fourier series 
expansion of the EM field in the $\phi$-direction represents the transformation of the EM field into spectral domain):

(a) in spatial domain

$$
\begin{aligned}
\overline{\bar{Y}}(\phi) & \cdot\left[\sum_{m=-\infty}^{\infty}\left(\tilde{\mathbf{E}}^{\text {inc }}\left(\rho_{\text {meta }}, m\right)+\tilde{\mathbf{E}}^{\text {scat }}\left(\rho_{\text {meta }}, m\right)\right) \mathrm{e}^{-\mathrm{j} m \phi}\right] \\
& =\hat{\rho} \times \sum_{m=-\infty}^{\infty} \Delta \tilde{\mathbf{H}}\left(\rho_{\text {meta }}, m\right) \mathrm{e}^{-\mathrm{j} m \phi},
\end{aligned}
$$

(b) in spectral domain

$$
\begin{aligned}
& \sum_{m^{\prime}=-\infty}^{\infty} \tilde{\tilde{\bar{Y}}}\left(m-m^{\prime}\right) \cdot\left(\tilde{\mathbf{E}}^{\text {inc }}\left(\rho_{\text {meta }}, m^{\prime}\right)+\tilde{\mathbf{E}}^{\text {scat }}\left(\rho_{\text {meta }}, m^{\prime}\right)\right) \\
& \quad=\hat{\rho} \times \Delta \tilde{\mathbf{H}}\left(\rho_{\text {meta }}, m\right) .
\end{aligned}
$$

Although both approaches are mathematically equivalent, they are quite different from a numerical implementation point of view, mostly due to presence of convolution summation/integration. We found it easier to implement the boundary conditions by using the spatial domain approach, in particular for structures that are non-homogeneous in axial direction.

Two analysis approaches will be presented here: Mode Matching (MM) and Method of Moments (MoM). The key difference between them is in the form they state the boundary conditions. These boundary conditions then constitute the basis for the analysis. Figure 2 illustrates the approaches, and they are further explained in the rest of this section.

In the MM approach, the unknowns are the amplitudes of field components in each region of interest. It is sufficient to consider the amplitudes of $E_{\mathrm{z}}$ and $H_{\mathrm{z}}$ components, which leads to the decomposition of the EM field on $\mathrm{TM}_{\mathrm{z}}$ and $\mathrm{TE}_{\mathrm{z}}$ modes. In the considered basic $2 \mathrm{D}$ case, the unknown amplitudes are $\alpha_{m}{ }^{(1)}$ and $\alpha_{m}{ }^{(2)}$, defined by (4.a) and (5.a). Now, having fulfilled the boundary conditions that state that the tangential E-field is continuous at the metasurface boundary, and the boundary condition (6), we arrive at the following system of equations:

$$
\begin{aligned}
& \alpha_{m}^{(1)} J_{m}\left(k_{0} \rho_{\text {meta }}\right)=\alpha_{m}^{(2)} H_{m}^{(2)}\left(k_{0} \rho_{\text {meta }}\right), \quad \forall m \in \mathbb{Z}, \\
& Y_{\mathrm{zz}}(\phi) \cdot\left[\begin{array}{l}
-\frac{k_{0} \eta_{0}}{4} H_{0}^{(2)}\left(k_{0} \rho_{\text {meta }}\right) I_{0} \\
+\frac{1}{2 \pi} \sum_{m=-\infty}^{\infty} \alpha_{m}^{(2)} H_{m}^{(2)}\left(k_{0} \rho_{\text {meta }}\right) \mathrm{e}^{-\mathrm{j} m \phi}
\end{array}\right] \\
& =\left(\frac{-\mathrm{j}}{\eta_{0}}\right) \frac{1}{2 \pi} \sum_{m=-\infty}^{\infty}\left(\alpha_{m}^{(2)} H_{m}^{(2)^{\prime}}\left(k_{0} \rho_{\text {meta }}\right)-\alpha_{m}^{(1)} J_{m}^{\prime}\left(k_{0} \rho_{\text {meta }}\right)\right) \mathrm{e}^{-\mathrm{j} m \phi} \text {. }
\end{aligned}
$$

Here $Y_{\mathrm{zz}}(\phi)$ is the zz-component of sheet admittance tensor $\overline{\bar{Y}}(\phi)$. If we determine the limit in summation as $M_{\max }$, we need to select $2 M_{\max }+1$ observation points to obtain a linear system of $2 M_{\max }+1$ equations with the same number of unknowns:

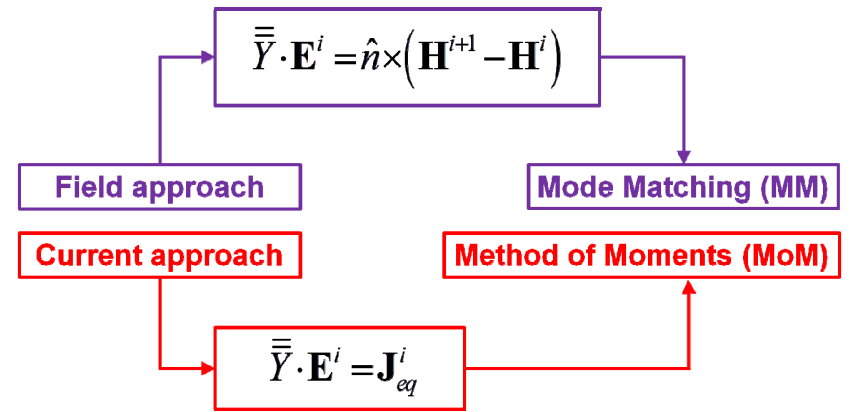

Fig. 2. Structure of two analysis approaches of curved metasurfaces.

$$
\begin{gathered}
Y_{\text {zz }}\left(\phi_{n}\right) \cdot\left[-\frac{k_{0} \eta_{0}}{4} H_{0}^{(2)}\left(k_{0} \rho_{\text {meta }}\right) I_{0}\right. \\
\left.+\frac{1}{2 \pi} \sum_{m=-M_{\max }}^{M_{\max }} \alpha_{m}^{(2)} H_{m}^{(2)}\left(k_{0} \rho_{\text {meta }}\right) \mathrm{e}^{-\mathrm{j} m \phi_{n}}\right] \\
=\left(\frac{-2}{\pi \eta_{0} k_{0} \rho_{\text {meta }}}\right) \frac{1}{2 \pi} \sum_{m=-\infty}^{\infty} \alpha_{m}^{(2)} \frac{1}{J_{m}\left(k_{0} \rho_{\text {meta }}\right)} \mathrm{e}^{-\mathrm{j} m \phi_{n}}, \\
n=1, \ldots, 2 M_{\text {max }}+1 .
\end{gathered}
$$

In this equation the connection between $\alpha_{m}{ }^{(1)}$ and $\alpha_{m}{ }^{(2)}$ (given by (8.a)), and the Wronskian expression for Bessel functions $J_{m}^{\prime}(x) H_{m}^{(2)}(x)-J_{m}(x) H_{m}^{(2) \prime}(x)=\mathrm{j} 2 / \pi x$ is implemented. The minimum number for $M_{\max }$ is $\operatorname{int}\left(k_{0} \rho_{\text {meta }}\right)$, as obtained from the far-field limit [21]. More accurate results are obtained with $M_{\max }=2 \operatorname{int}\left(k_{0} \rho_{\text {meta }}\right)$, and this is the limit we used in calculations. Usually, one observation point is located at the center of each metasurface cell. However, if the selected $M_{\max }$ is large, more than one observation point can be located on each metasurface cell.

Generalization of the MM approach to the multilayer case is straightforward - one should assume the representation of $E_{\mathrm{z}}$ and $H_{\mathrm{z}}$ components in each region as

$$
\begin{gathered}
\tilde{E}_{\mathrm{z}}^{i}(\rho, m)=C_{1 m}^{i} J_{m}\left(k_{i} \rho\right)+C_{2 m}^{i} H_{m}^{(2)}\left(k_{i} \rho\right), \\
\tilde{H}_{\mathrm{z}}^{i}(\rho, m)=C_{3 m}^{i} J_{m}\left(k_{i} \rho\right)+C_{4 m}^{i} H_{m}^{(2)}\left(k_{i} \rho\right) .
\end{gathered}
$$

Here, we used standing wave and outward travelling cylindrical wave representation of the field in each layer, with $k_{i}$ being the wave number of $i$-th layer, and $C_{m}^{i}$ being the wave amplitudes that need to be determined. The other field components are determined using the following expressions:

$$
\begin{gathered}
\tilde{E}_{\phi}^{i}(\rho, m)=\frac{\mathrm{j} \eta_{n}}{k_{n}} \frac{\partial}{\partial \rho} \tilde{H}_{z}^{i}(\rho, m), \\
\tilde{H}_{\phi}^{i}\left(\rho, m, k_{z}\right)=-\frac{\mathrm{j}}{\eta_{n} k_{n}} \frac{\partial}{\partial \rho} \tilde{E}_{z}^{i}\left(\rho, m, k_{z}\right) .
\end{gathered}
$$

The boundary conditions at each boundary $\rho_{i}$, connecting the field representation in neighboring regions are:

$$
\tilde{\mathbf{E}}^{i}\left(\rho_{\text {meta }}^{i}, m\right)=\tilde{\mathbf{E}}^{i+1}\left(\rho_{\text {meta }}^{i}, m\right), \quad \forall m \in \mathbb{Z},
$$




$$
\begin{gathered}
\overline{\bar{Y}}\left(\rho_{\text {meta }}^{i}, \phi\right) \cdot\left[\sum_{m=-\infty}^{\infty}\left(\tilde{\mathbf{E}}^{\text {inc }}\left(\rho_{\text {meta }}^{i}, m\right)+\tilde{\mathbf{E}}^{\text {scat }, i}\left(\rho_{\text {meta }}^{i}, m\right)\right) \mathrm{e}^{-\mathrm{j} m \phi}\right] \\
=\hat{\rho} \times \sum_{m=-\infty}^{\infty}\left(\tilde{\mathbf{H}}^{i+1}\left(\rho_{\text {meta }}^{i}, m\right)-\tilde{\mathbf{H}}^{i}\left(\rho_{\text {meta }}^{i}, m\right)\right) \mathrm{e}^{-\mathrm{j} m \phi} .
\end{gathered}
$$

The Method of Moment (MoM) procedure is based on the introduction of induced surface electric current $\mathbf{J}_{\mathrm{av}}$ and approximating it by a set of base functions $\mathbf{J}^{p}$ av with unknown amplitudes $\alpha_{p}$ :

$$
\mathbf{J}_{\text {av }}\left(\rho_{\text {meta }}^{i}, \phi\right)=\hat{\rho} \times\left(\Delta \mathbf{H}\left(\rho_{\text {meta }}^{i}, \phi\right)\right)=\sum_{p} \alpha_{p} \mathbf{J}_{\text {av }}^{p}\left(\rho_{\text {meta }}^{i}, \phi\right) .
$$

It is sufficient to consider only one boundary condition per metasurface layer:

$$
\overline{\bar{Y}}\left(\rho_{\text {meta }}^{i}, \phi\right) \cdot \mathbf{E}\left(\rho_{\text {meta }}^{i}, \phi\right)=\mathbf{J}_{\text {av }}\left(\rho_{\text {meta }}^{i}, \phi\right) .
$$

By implementing the Galerkin approach, the final system of MoM equations has a form:

$$
\begin{gathered}
\int_{L_{q}} \mathbf{J}_{\text {av }}^{q}\left(\rho_{\text {meta }}^{j}, \phi\right) \cdot\left[\overline { \overline { Y } } ( \rho _ { \text { meta } } ^ { j } , \phi ) \cdot \left[\mathbf{E}^{\text {inc }}\left(\rho_{\text {meta }}^{j}, \phi\right)\right.\right. \\
\left.\left.+\frac{1}{2 \pi} \sum_{m=-M_{\text {max }}}^{M_{\text {max }}} \sum_{p=1}^{N_{\text {Mou }}} \alpha_{p} \tilde{\bar{G}}^{\mathrm{EJ}}\left(m, \rho_{\text {meta }}^{j} \mid \rho_{\text {meta }}^{i}\right) \cdot \tilde{\mathbf{J}}_{\text {av }}^{p}\left(\rho_{\text {meta }}^{i}, m\right) \mathrm{e}^{-\mathrm{j} m \phi}\right]\right] \mathrm{d} \phi \\
=\int_{L_{q}} \mathbf{J}_{\text {av }}^{q}\left(\rho_{\text {meta }}^{j}, \phi\right) \cdot\left[\sum_{p=1}^{N_{\text {MoM }}} \alpha_{p} \mathbf{J}_{\text {av }}^{p}\left(\rho_{\text {meta }}^{i}, \phi\right)\right] \mathrm{d} \phi, \\
q=1, \ldots, N_{\text {MoM }} .
\end{gathered}
$$

Here, the presence of a supporting dielectric structure is taken into account through the $\tilde{\overline{\bar{G}}}^{\mathrm{EJ}}$ Green's function for a multilayer cylindrical structure. Its spectral domain form is easily calculated by available algorithms, such as G1DMULT [22], [23]. For a basic cylindrical structure from Fig. 1(b) the Green's function is

$$
\begin{aligned}
\tilde{G}_{\text {zz }}^{\mathrm{EJ}}\left(m, \rho \mid \rho_{\text {meta }}\right)=-\frac{\pi \eta_{0} k_{0}}{2} \rho_{\text {meta }} \\
\cdot\left\{\begin{array}{ccc}
H_{m}^{(2)}\left(k_{0} \rho_{\text {meta }}\right) & J_{m}\left(k_{0} \rho\right) & \rho \leq \rho_{\text {meta }} \\
J_{m}\left(k_{0} \rho_{\text {meta }}\right) & H_{m}^{(2)}\left(k_{0} \rho\right) & \rho \geq \rho_{\text {meta }}
\end{array}\right.
\end{aligned}
$$

Note that, by selecting specific basis and test functions (namely Fourier series basis functions and point- matching test functions), both approaches (MM and MoM) will lead to identical linear system of equations. Furthermore, note that the presented 2D formulation can easily be generalized for a 3D problem by implementing the Fourier transformation in $z$-direction to model the spatial variation of metasurface admittance in axial direction.

\section{Results}

Similarly as in previous section, a cylindrical metasurface placed around an axial incident field source is considered. The EM field source is exciting an omnidirectional radiation pattern in the radial plane. The purpose of the metasurface is to modify the incident field so that two main beams are formed in the region outside the metasurface, angularly displaced by $90^{\circ}$, and symmetrical to the central normal plane of the metasurface structure. The considered single-layer metasurface is printed on a thin substrate $\left(\varepsilon_{\mathrm{r}}=2.55, h=0.13 \mathrm{~mm}\right)$. It contains 24 inductive cells that are in practice realized using stripes or meander lines. The radius of the cylinder is $6 \mathrm{~cm}$ and the width of each cell is $7.854 \mathrm{~mm}$. Only half of the cylinder is covered by the metasurface structure. The variation of surface sheet impedance is listed in Tab. 1, and the practical realization is shown in Fig. 3. The prototype was developed using standard PCB technology. The used thin PCB substrate was flexible enough to bend the PCB structure into a halfcylinder form of the desired radius.

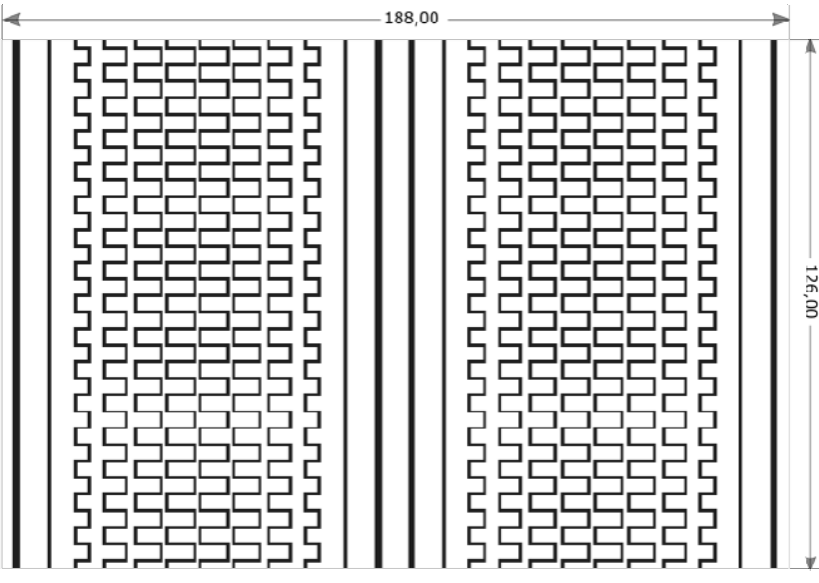

Fig. 3. PCB realization of metasurface layer featuring stripes and meander lines.

\begin{tabular}{|c|c|c|c|c|c|}
\hline $\begin{array}{c}\text { Segment } \\
\text { No. }\end{array}$ & $\begin{array}{c}\text { Type of metasurface } \\
\text { structure }\end{array}$ & Stripe width & $\begin{array}{c}\text { Total width of } \\
\text { meander line }\end{array}$ & $\begin{array}{c}\text { Calculated surface } \\
\text { sheet impedance }\end{array}$ & $\begin{array}{c}\text { Estimated surface sheet } \\
\text { impedance for conductive } \\
\text { paint realization }\end{array}$ \\
\hline 1 & stripes & $1.3 \mathrm{~mm}$ & - & $\mathrm{j} \cdot 126.16 \Omega$ & $0.3+\mathrm{j} \cdot 126.16 \Omega$ \\
\hline 2 & stripes & $0.5 \mathrm{~mm}$ & - & $\mathrm{j} \cdot 223.66 \Omega$ & $0.8+\mathrm{j} \cdot 223.66 \Omega$ \\
\hline 3 & meander line & $0.5 \mathrm{~mm}$ & $3.5 \mathrm{~mm}$ & $\mathrm{j} \cdot 392.53 \Omega$ & $2.2+\mathrm{j} \cdot 392.53 \Omega$ \\
\hline 4 & meander line & $0.5 \mathrm{~mm}$ & $5.15 \mathrm{~mm}$ & $\mathrm{j} \cdot 587.52 \Omega$ & $2.8+\mathrm{j} \cdot 587.52 \Omega$ \\
\hline 5 & meander line & $0.5 \mathrm{~mm}$ & $6.1 \mathrm{~mm}$ & $\mathrm{j} \cdot 756.39 \Omega$ & $3.1+\mathrm{j} \cdot 756.39 \Omega$ \\
\hline 6 & meander line & $0.5 \mathrm{~mm}$ & $6.5 \mathrm{~mm}$ & $\mathrm{j} \cdot 853.89 \Omega$ & $3.2+\mathrm{j} \cdot 853.89 \Omega$ \\
\hline
\end{tabular}

Tab. 1. Details of the design of single-layer cylindrical metasurface structure containing 24 segments: straight stripes have a variable line width and meander lines have a fixed line width and a variable total width. The radius of the cylindrical metasurface structure is $60 \mathrm{~mm}$ and the central working frequency is $10 \mathrm{GHz}$. Due to symmetry, only $1 / 4$ of the structure is described. 
For additional verification, the metasurface structure was also simulated using CST Microwave Studio. In it, we analyzed the whole structure with all the details, which required computing time of several hours, compared to a few seconds needed by the in-house developed code using the presented analysis approach. A comparison between the measured radiated field and calculated results are given in Fig. 4.

The production of single-curved structures (i.e. structures with one principal direction of curvature) is of the same order of complexity as the production of their planar counterparts, and standard printed circuit board (PCB) production technology can still be used to a large extent. However, there are several practical details to be considered. First, by bending a planar substrate, the length of the outer surface becomes larger than the length of the inner surface. This extension of the outer surface length should be considered while designing the metasurface elements. Next, when designing structures with more than two metasurface layers, it is important to adhesively bond the layers together without introducing air pockets, gaps and cracks between them. Therefore, in this process it is important to have a rigid frame (i.e. mould) to successfully bend and glue layers that form a multilayer structure together. Finally, it is important to choose a substrate suitable for bending, i.e. to select a microwave substrate with a low value of flexural modulus.

Double-curved metasurface structures are much harder to produce. It is not possible to just bend the substrate material, since we now have two principal orthogonal directions of curvature. One possibility is to produce the dielectric supporting structure using additive manufacturing technology. The metallization process is more complex, and we have investigated several options for performing metallization: the first using sprays with conductive paint, the other using electroless copper plating process, and finally using sputtering technology.

As it proves to be the simplest approach, the first option was tested by repeatedly producing a single-layer

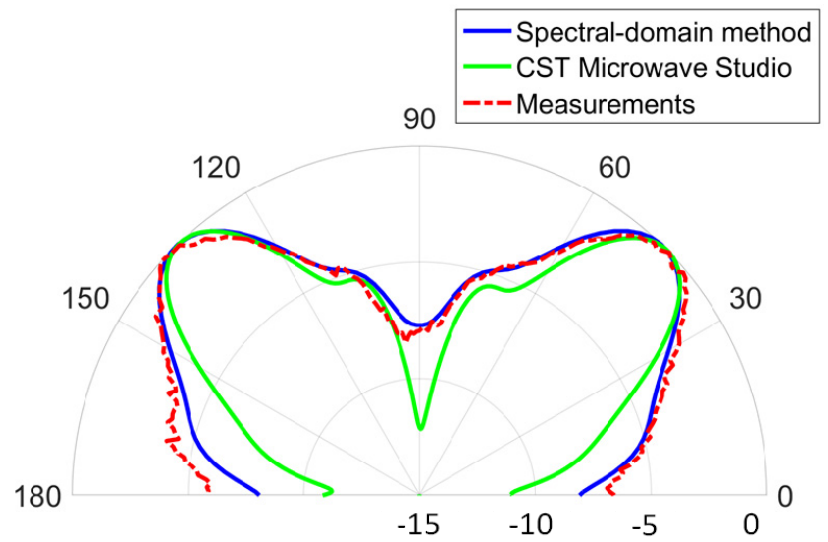

Fig. 4. Electric field response of a curved metasurface printed on a thin supporting dielectric structure. Comparison between measurements, and calculations obtained from the developed spectral-domain method and CST Microwave Studio.

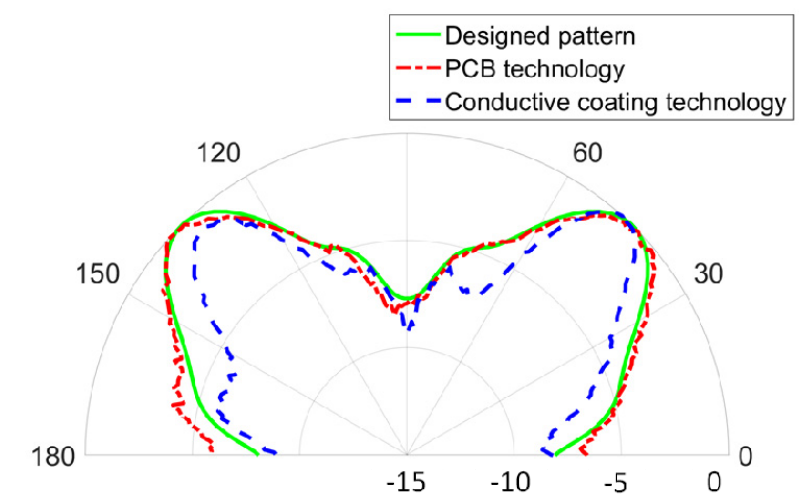

Fig. 5. Electric field response of a curved metasurface printed on a thin supporting structure. Comparison of measured results between two metasurface structures; one produced using standard PCB production technology, and the other produced by spraying the Conductive silver coating 3830 paint.

metasurface shown in Fig. 3. First, a template for laying the spray-paint was prepared out of solid thin cardboard. For precise cutting we used a laser-equipped $\mathrm{CNC}$ machine. Next, the conductive spray-paint was applied on the dielectric substrate. Conductive silver coating 3830 paint, produced by the Hollandshielding Company, was used. The declared surface resistance is better than $0.015 \Omega /$ square at 25 microns coating thickness. We tried to estimate the losses in stripes and meander lines of the produced prototype. To do that, we started with an approximate formula for surface impedance of periodic stripes with included losses [24]:

$$
\left(Y_{\mathrm{zz}}^{\text {approx }}\right)^{-1}=R_{\sigma} P_{\phi}+\mathrm{j} \frac{k_{0} \eta_{0}}{2 \pi} P_{\phi} \log \left(\csc \left(\frac{\pi W}{2 P_{\phi}}\right)\right) .
$$

Here, $P_{\phi}$ is the period of the stripes, $W$ is the stripe width, and $R_{\sigma}$ is the term that represents the resistance per unit length. We estimated $R_{\sigma}$ by measuring the DC resistance, and the obtained values are also given in Tab. 1. It is found that paint of large conductivity introduces negligible additional losses, i.e. the realized gain pattern remains almost unaffected. The comparison of measured radiation patterns between two structures, the one produced by standard PCB production technology, and the other produced using conductive paint approach, is given in Fig. 5. Both curves in Fig. 5 are normalized to the maximum of the measured gain, achieved with the standard PCB case. The goal of forming two main beams at angles $\pm 45^{\circ}$ is achieved. While no additional losses were introduced by the conductive paint coating, we did not manage to keep the precision of $\mathrm{PCB}$ production, so the shape of the radiation pattern is somewhat perturbed, as compared to both standard PCB production technique, and the desired radiation pattern.

\section{Conclusion}

The paper discusses a spectral-domain approach for analyzing cylindrical cascaded metasurface structures with 
spatially-varying distribution of surface sheet impedance. The formulation also covers metasurfaces that are located only on a part of canonical curved surface. Although the spectral-domain approach is selected due to inherently convenient EM field representation for multilayer cylindrical structure, several different formulations of the analysis approach are discussed, and the selection of the most suitable depends on the domain (spectral or spatial) in which we would like to calculate the surface sheet impedance distribution and on the unknown quantity that needs to be determined - be it the EM field in each cylindrical layer or the induced current distribution at each curved metasurface sheet.

The problem of production of curved metasurface structures is also discussed in the paper. Single-curved structures (i.e. structures with one principal direction of curvature) can be quite easily produced using standard printed circuit board (PCB) technology; one just needs to select a proper substrate suitable for bending. However, double-curved structures are much harder to produce. One possibility is to produce the supporting dielectric structure using e.g. additive $3 \mathrm{D}$ printing technology and then to metalize the desired patterns onto it using conductive paint. There are still many technological issues to be solved, in particular, the problem of fine control of metallic pattern dimensions, and building curved multilayer metasurface structures without cracks and gaps between layers. These are the topics for future investigations.

\section{Acknowledgments}

This work was supported in part by the Air Force Office of Scientific Research, Air Force Material Command, USAF under Award No. FA9550-15-1-0121, and Ericsson Nikola Tesla d.d. and University of Zagreb Faculty of Electrical Engineering and Computing under the EWITA project.

\section{References}

[1] HOLlOWAY, C. L., KUESTER, E.F., GORDON, J., et al. An overview of the theory and applications of metasurfaces: The twodimensional equivalents of metamaterials. IEEE Antennas and Propagation Magazine, 2012, vol. 54, no. 2, p. 10-35. DOI: 10.1109/MAP.2012.6230714

[2] PFEIFFER C., GRBIC, A. Bianisotropic metasurfaces for optimal polarization control: Analysis and synthesis. Physical Review Applied, 2014, vol. 2, no. 4, p. 1-11. DOI: 10.1103/PhysRevApplied.2.044011

[3] PFEIFFER C., GRBIC, A. Metamaterial Huygens surfaces: Tailoring wave fronts with reflectionless sheets. Physical Review Letters, 2013, vol. 110, no. 19, p. 1-5. DOI: 10.1103/PhysRevLett.110.197401

[4] MUNK, B. A. Frequency Selective Surfaces: Theory and Design. John Wiley\& Sons, 2005. ISBN: 978-0471370475
[5] MACI, S., MINATTI, G., CASAlETTI, M., et al. Metasurfing: Addressing waves on impenetrable metasurfaces. IEEE Antennas and Wireless Propagation Letters, 2011, vol. 10, p. 1499-1502. DOI: $10.1109 /$ LAWP.2012.2183631

[6] KUESTER, E. F., MOHAMED, M. A., PIKET-MAY, M., et al. Averaged transition conditions for electromagnetic fields at a metafilm. IEEE Transactions on Antennas and Propagation, 2003, vol. 51, no. 10, p. 2641-2651. DOI: 10.1109/TAP.2003.817560

[7] ZHAO, Y., BELKIN, M. A., ALÙ, A. Twisted optical metamaterials for planarized ultrathin broadband circular polarizers. Nature Communications, 2012, vol. 3, p. 870-876. DOI: $10.1038 /$ ncomms 18

[8] PFEIFFER, C., GRBIC, A. Cascaded metasurfaces for complete phase and polarization control. Applied Physics Letters, 2013, vol. 102, no. 23, p. 1-4. DOI: 10.1063/1.4810873

[9] MONTICONE, F., ESTAKHRI, N. M., ALÙ, A. Full control of nanoscale optical transmission with a composite metascreen. Physical Review Letters, 2013, vol. 110, p. 1-5. DOI:10.1103/PhysRevLett.110.203903

[10] NIEMI, T., KARILAINEN, A., TRETYAKOV, S. Synthesis of polarization transformers. IEEE Transactions on Antennas and Propagations, 2013, vol. 61, no. 6, p. 3102-3111. DOI: 10.1109/TAP.2013.2252136

[11] SElvanAyagam, M., Eleftheriades, G.V. Polarization control using tensor Huygens surfaces. IEEE Transactions on Antennas and Propagation, 2014, vol. 62, no. 12, p. 6155-6168. DOI: 10.1109 /TAP.2014.2359208

[12] PATEL, A. M., GRBIC, A. Transformation electromagnetics devices based on printed-circuit tensor impedance surfaces. IEEE Transactions on Microwave Theory and Techniques, 2014, vol. 62, no. 5, p. 1102-1111. DOI: 10.1109/TMTT.2014.2314440

[13] ELEK, F., TIERNEY, B. B., GRBIC, A. Synthesis of printedcircuit tensor impedance surfaces controlling phase and power flow. IEEE Transactions on Antennas and Propagations, 2015, vol. 63, no. 9, p. 3956-3962. DOI: 10.1109/TAP.2015.2448234

[14] RAEKER, B. O., RUDOLPH, S. M. Arbitrary transformation of antenna radiation using a cylindrical impedance metasurface. IEEE Antennas and Wireless Propagation Letters, 2016, vol. 15, p. 1101-1104. DOI: 10.1109/LAWP.2015.2494739

[15] RAEKER, B. O., RUDOLPH, S. M. Verification of arbitrary radiation pattern control using a cylindrical impedance metasurface. IEEE Antennas and Wireless Propagation Letters, 2017, vol. 16, p. 995-998. DOI: 10.1109/LAWP.2016.2616106

[16] CHEN, P.-Y., ALÙ, A. Mantle cloaking using thin patterned metasurfaces. Physical Review B, 2011, vol. 84, p. 1-13. DOI: 10.1103/PhysRevB.84.205110

[17] PADOORU, Y. R., YAKOVLEV, A. B., CHEN, P.-Y., et al. Linesource excitation of realistic conformal metasurface cloaks. Journal of Applied Physics, 2012, vol. 112, no. 10, p. 1-11. DOI: $10.1063 / 1.4765688$

[18] SORIC, J. C., MONTI, A., TOSCANO, A., et al. Dual-polarized reduction of dipole antenna blockage using mantle cloaks. IEEE Transactions on Antennas and Propagation, 2015, vol. 63, no. 11, p. 4827-4834. DOI: 10.1109/TAP.2015.2476468

[19] VElluCCI, S., MONTI, A., TOSCANO, A., et al. Scattering manipulation and camouflage of electrically small objects through metasurfaces. Physical Review Applied, 2017, vol. 7, p. 1-12. DOI: 10.1103/PhysRevApplied.7.034032

[20] SIPUS, Z., BOSILJEVAC, M., GRBIC, A. Modelling cascaded cylindrical metasurfaces using sheet impedances and a transmission matrix formulation. IET Microwaves, Antennas and Propagation, 2018, vol. 12, no. 7, p. 1041-1047. DOI: 10.1049/iet-map.2017.0465 
[21] HANSEN, J. E. (ed.) Spherical Near-Field Antenna Measurements. Stevenage (U.K.): Peregrinus, 1988. ISBN: 9780863411106

[22] SIPUS, Z., KILDAL, P.-S., LEIJON, R., et al. An algorithm for calculating Green's functions for planar, circular cylindrical and spherical multilayer substrates. Applied Computational Electromagnetics Society Journal, 1998, vol. 13, no. 3, p. 243-254.

[23] KILDAL, P.-S., SIPUS, Z., YANG, J., et al. Useful physical images and algorithms for vector dyadic Green's functions in 3D spatial, 2D spectral and 1D spectral domains for solving multilayer and multiregion field problems. IEEE Antennas and Propagation Magazine, 2017, vol. 59, no. 4, p. 106-116. DOI: 10.1109/MAP.2017.2706665

[24] TRETYAKOV, S. A. Analytical Modeling in Applied Electromagnetics. Norwood: Artech House, 2003. ISBN: 978-1630812836

\section{About the Authors ...}

Zvonimir ŠIPUŠ was born in Zagreb, Croatia, in 1964. He received the B.Sc. and M.Sc. degrees in Electrical Engineering from the University of Zagreb, Croatia, in 1988 and 1991, respectively, and the Ph.D. degree in Electrical Engineering from the Chalmers University of Technology, Gothenburg, Sweden, in 1997. The same year he joined the Faculty of Electrical Engineering and Computing, University of Zagreb, where he is currently a Professor. From
1999 to 2005, he was an Adjunct Researcher with the Department of Electromagnetics, Chalmers University of Technology. Since 2006, he has been involved in teaching with the European School of Antennas. His current research interests include analysis and design of electromagnetic structures with application to antennas, microwaves, and optical communication and sensor systems.

Zoran EREŠ was born in Zagreb, Croatia, in 1975. He received the M.Sc. and Ph.D. degrees in Electrical Engineering from the University of Zagreb, Croatia, in 2002 and 2019, respectively, Since 2002, he has been with the Rudjer Boskovic Institute, Zagreb, as an expert associate. His research interests include design and experimental realization of analog and digital electronic circuits, vacuum techniques, CVD synthesis of graphene and its application in electronic devices.

Dominik BARBARIĆ was born in Zagreb, Croatia, in 1993. He received his B.Sc. degree in 2015, and his M.Sc. degree in 2017, both from the Faculty of Electrical Engineering and Computing, University of Zagreb. He is pursuing a $\mathrm{PhD}$ degree at the same University, and his research interests include transmitter frontends and antenna arrays. He is currently employed at Ericsson Nikola Tesla d.d., where he's working on joint research projects with the University of Zagreb, and the development of 5G radio base stations. 